\title{
Inhibitory potential of Lactobacillus species isolated from fermented dairy products against Escherichia coli and Staphylococcus aureus
}

\author{
Sukhminderjit Kaur, Madhu Sharma \\ Department of Biotechnology \\ Shaheed Udham Singh College of Research and Technology, Tangori, Mohali, India
}

\begin{abstract}
Background \& Objectives: Probiotics exert a strong antagonistic activity against many microorganisms including food spoilage organisms and enteropathogens. Lactobacilli find increasing acceptance as probiotics by showing its beneficial effects. Keeping in view the benefits of probiotics in literature, the present study was planned to isolate Lactobacillus spp. from different fermented dairy products and to study the inhibitory potential against biofilm forming bacteria viz. Escherichia coli (E. coli) and Staphylococcus aureus (S. aureus).
\end{abstract}

Methods: Lactobacillus spp. were isolated from different fermented dairy products and characterized. The antimicrobial activity of each Lactobacillus spp. was checked by agar well diffusion and overlay methods. The strains showing inhibitions in these assays were further used to detect biofilm inhibition against $E$. coli and $S$. aureus by microtiter plate biofilm assay method.

Results: A total of 21 samples were studied of which pure growth was observed in $67 \%$ and mixed growth was observed in $33 \%$ of the samples. Of 18 isolates obtained, $45 \%$ belonged to Lactobacillus plantarum (L. plantarum), $33 \%$ were Lactobacillus acidophilus (L. acidophilus) and $22 \%$ comprised of Lactobacillus fermentum (L. fermentum). Ten isolates showed inhibition against E.coli and 9 isolates showed inhibitory activity against $S$. aureus in agar well diffusion assay and overlay method. In microtiter plate biofilm assay, the absorbance values were less in the wells where E. coli and S. aureus were mixed with the Lactobacillus broth.

Interpretation and Conclusions: The Lactobacillus strains showing inhibitory activities against the pathogens can be used as probiotics or as starter culture in food fermentations after confirming other attributes.

Keywords: Bacteriocins, Biofilm, Lactobacillus, Probiotics

\section{INTRODUCTION}

Probiotic bacteria are well known to have a positive effect on the maintenance of human health. Lactobacilli find increasing acceptance as probiotics which aid in stimulating immune responses, preventing infections by pathogenic bacteria, treating and preventing diarrhoea. ${ }^{1}$ They can improve lactose digestion, play a role in preventing and treating diarrhea, helping the body to resist and fight infections. ${ }^{2}$

\section{Corresponding Author :}

Dr. Sukhminderjit Kaur,

Assistant Professor

Department of Biotechnology

Shaheed Udham Singh College of Research and

Technology, Tangori, Mohali. Pin: 140306

Email: sukhminderphd_6@yahoo.co.in
Probiotics exert a strong antagonistic activity against many microorganisms including food spoilage organisms and enteropathogens. Probiotics are being tried as alternative to antibiotics in treating many gastrointestinal diseases. ${ }^{3}$ Probiotics exert their beneficial effects by producing various compounds such as organic acids, hydrogen peroxide, diacetyl, bacteriocins and adhesion inhibitors such as biosurfactants. ${ }^{4}$ Lactobacilli are important in the food and dairy industries because lactic acid and other organic acids produced by these bacteria act as natural preservatives as well as flavor enhancers.

Bacteriocins are extracellularly released proteinaceous antimicrobial compounds released which exhibit bactericidal effect against closely related bacteria. ${ }^{5}$ Biosurfactant production by these bacteria can prevent biofilm formation by bacterial pathogens. ${ }^{6}$ Lactobacillus spp. exhibiting antagonistic activities towards food spoilage bacteria or enteropathogens are especially 
important as these microorganisms even at low levels pose a significant spoilage and public health threat. ${ }^{7}$ Considering the beneficial effects of probiotics, the present study was planned to explore the inhibitory potential of Lactobacillus spp. isolated from fermented dairy products for their inhibitory potential against two biofilm forming microorganisms viz. Escherichia coli (E. coli) and Staphylococcus aureus (S. aureus).

\section{MATERIAL AND METHODS}

Lactobacillus species isolated from different fermented dairy products like curd, cheese, butter, fermented milk, lassi and milk were randomly collected from retail markets of Chandigarh, India and its periphery.

Isolation of Lactic Acid Bacteria: Lactobacillus spp. were isolated from various fermented dairy samples using 10 fold serial dilution. One $\mathrm{ml}$ of each dilution was plated on de-Mann Rogosa and Sharpe (MRS) agar (HiMedia, Mumbai) plate. Then plates were incubated at $37^{\circ} \mathrm{C}$ for $48 \mathrm{hrs}$. After $48 \mathrm{hrs}$ of incubation, the plates were checked for colony, color, size, morphology and texture. The isolated colonies were purified by streak inoculation on MRS agar plates and purified culture were stored on MRS agar slants and kept at $4^{\circ} \mathrm{C}$ till further use. Gram staining was performed on the purified isolates for determining the cell morphology by standard procedure. Biochemical characterization of isolates was done carrying out catalase test, arginine hydrolysis, nitrate reduction, motility, sugar fermentation (glucose, sucrose and dextrose) and gas formation tests. ${ }^{8}$

Antimicrobial activity detection assay: Anti bacterial activity of the Lactobacillus spp. was determined by agar well diffusion and overlay method as described earlier. $^{9}$

Agar well diffusion method: The antimicrobial activity due to bacteriocin produced by Lactobacillus spp. in broth against $E$. coli and $S$. aureus was checked by agar well diffusion assay. Lactobacillus culture was grown in MRS broth for $18-24 \mathrm{hrs}$ at $37^{\circ} \mathrm{C}$. The cells were removed by centrifugation at $12000 \mathrm{rpm}$ for $20 \mathrm{~min}$ at $5^{\circ} \mathrm{C}$. The culture supernatant thus obtained was sterilized by passing through a sterile $0.22 \mu \mathrm{m}$ membrane filter. The supernatant was adjusted to $\mathrm{pH}$ of 7 by addition of $1 \mathrm{~N} \mathrm{HCl}$ and $1 \mathrm{~N} \mathrm{NaOH}$ and used in the assay.

Mueller Hinton agar (HiMedia, Mumbai) plates were inoculated with $0.1 \mathrm{ml}$ of $24 \mathrm{hrs}$ old culture containing approximately $10^{8} \mathrm{CFU} / \mathrm{ml}$ of $S$. aureus or E. coli. Wells of $5 \mathrm{~mm}$ diameter were made in Mueller Hinton agar plates. Each well was filled with $100 \mu \mathrm{l}$ of Lactobacillus spp. culture supernatant. The inoculated plates were incubated at $37^{\circ} \mathrm{C}$ for $24 \mathrm{hrs}$. Zone of inhibition around the wells was recorded.

Overlay method: An overnight culture of Lactobacillus spp. was spotted onto the surface of MRS plate and incubated for $16 \mathrm{hrs}$ at $37^{\circ} \mathrm{C}$ to allow colonies to develop. Muller-Hinton agar inoculated with $0.1 \mathrm{ml}$ of $24 \mathrm{hrs}$ old culture containing approximately $10^{8} \mathrm{CFU} / \mathrm{ml}$ of $S$. aureus or E. coli was overlaid onto MRS agar. The plates were incubated at $37^{\circ} \mathrm{C}$ for $24 \mathrm{hrs}$. Zone of inhibition formed around the Lactobacillus culture spot was measured.

Inhibiton of biofilm formation by $E$. coli and $S$. aureus: The inhibition of biofilm formation by Lactobacillus spp. was studied by microtiter well plate biofilm assay. ${ }^{10}$ Each Lactobacillus isolate and indicator strains E. coli and $S$. aureus were inoculated in a 3-5 ml of MRS and nutrient broth respectively and grown to stationary phase. Lactobacillus cultures were centrifuged at $12000 \mathrm{rpm}$ for $20 \mathrm{~min}$ at $5^{\circ} \mathrm{C}$. The culture supernatant thus obtained was then sterilized by passing through a sterile $0.22 \mu \mathrm{m}$ membrane filter (Millipore). Microtiter plate which has not been tissue culture treated was taken. The first well of micro titer plate was filled with $100 \mu \mathrm{l}$ of distilled water and was taken as negative control. The second well of the plate was filled with $50 \mu \mathrm{l}$ of $E$. coli culture and $50 \mu \mathrm{l}$ of sterile distilled water and set as positive control. Then next ten wells of plate were filled with 50 $\mu \mathrm{l}$ of $E$. coli and $50 \mu \mathrm{l}$ of the various Lactobacillus spp. broth. The same procedure was repeated for $S$. aureus.

The microtiter plate was then covered and incubated at $37^{\circ} \mathrm{C}$ for $24 \mathrm{hrs}$. After incubation time, the planktonic bacteria were removed from each well and the wells were washed with sterile distilled water. Water was replaced when it became cloudy. Plate was washed three times. Then $125 \mu \mathrm{l}$ of $0.1 \%$ crystal violet solution was added to each well and the plate was kept at room temperature for $10 \mathrm{~min}$. After $10 \mathrm{~min}$, each well of microtiter plate was washed three times with sterile distilled water to remove crystal violet solution. After drying, $200 \mu \mathrm{l}$ of $95 \%$ ethanol was added to each stained well. The plate was incubated for 10 to $15 \mathrm{~min}$ at room temperature to solublize the dye. The contents of each well were mixed and then $125 \mu \mathrm{l}$ of the solution was transferred to another microtiter well plate. Optical density 


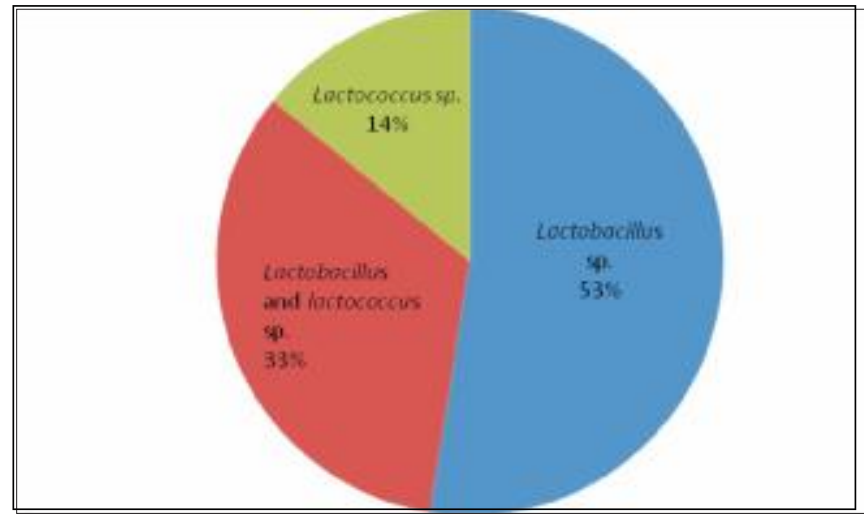

Figure I: Lactic acid bacteria isolated from fermented dairy products

of each well was taken at $600 \mathrm{~nm}$ using UV - Visible spectrophotometer (Systronics, India).

\section{RESULTS AND DISCUSSION}

A total of 21 different fermented dairy products were collected and processed for isolation of Lactobacillus spp. Pure growth was observed in $67 \%$ of the samples whereas mixed growth was observed in $33 \%$ of the samples. The 18 lactobacilli isolates were characterized on the basis of morphological and biochemical tests. Microscopic identification determined the presence of the rod shaped cells. On the basis of Gram's staining and catalase test it was found that the 18 rod shaped isolates were Gram positive and catalase negative which are the characteristics of lactobacilli. Out of 21 samples, pure growth of Lactobacillus spp. was isolated from 11 (53\%) samples, mixed growth from 7 (33\%) of the samples along with Lactococcus spp. whereas 3 (14\%) isolates were observed as pure Lactococcus spp. as shown in Figure I.

All isolates were found positive for nitrate reductase production and sucrose fermentation. Apart from this, the isolates which were negative for arginine hydrolysis and gas production were identified as Lactobacillus acidophilus (L. acidophilus). The isolates which were positive for arginine hydrolysis and negative for gas production were identified as Lactobacillus plantarum (L. plantarum) whereas the isolates showing arginine hydrolysis and gas production in sugar fermentation were identified as Lactobacillus fermentum (L. fermentum). On the basis of biochemical results, it was observed that among these isolates $45 \%$ were L. plantarum, $33 \%$ were L. acidophilus, and $22 \%$ were L. fermentum.

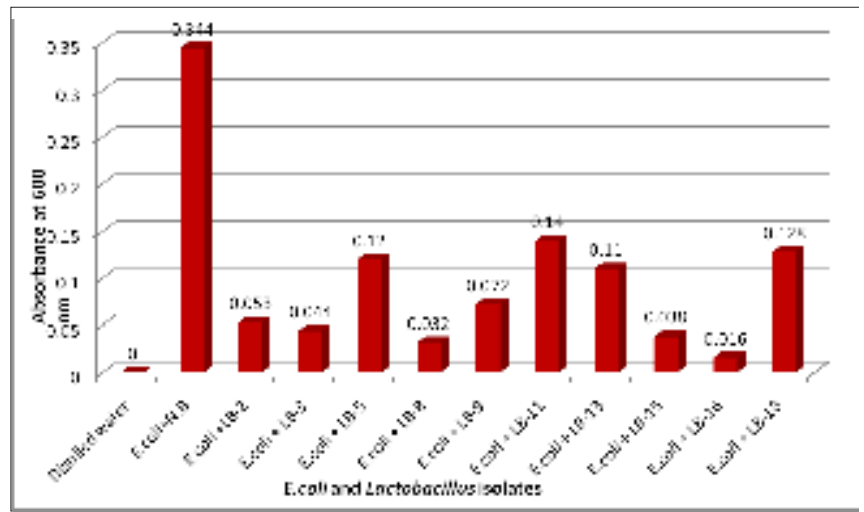

FigureII: Effect of Lactobacillus spp. on biofilm formation by E. coli

N.B = Nutrient broth, LB Lactobacillus

Lactobacillus strains have been isolated and characterized in many studies. Species belonging to $L$. acidophilus, L. plantarum and L. fermentum are frequently isolated from fermented food products. ${ }^{11,12}$

All the Lactobacillus spp. isolated were studied for antimicrobial activity against $E$. coli and $S$. aureus by agar well diffusion method and overlay method. The bacteriocin activity in agar well diffusion assay by Lactobacillus isolates is shown in Table I. Of the 18 isolates only $10(55 \%)$ isolates inhibited the growth of E.coli and $9(50 \%)$ showed inhibitory activity against $S$. aureus. Among the ten isolates, 5 isolates of $L$. plantarum (LB- 8, LB-9, LB-13, LB-16 and LB-18), 3 isolates of L. acidophilus (LB-5, LB-11, and LB-15), and 2 isolates of L. fermentum (LB-2, LB-3) showed inhibitory activity against $E$. coli (Figure II). Similarly 4 isolates of L. plantarum (LB-8, LB-9, LB-13, LB-16), 3 isolates of L. acidophillus (LB-5, LB-11, LB-15) and 2 isolates of L. fermentum (LB-2 and LB-3) showed inhibitory activity against $S$. aureus.

The antimicrobial activity of Lactobacillus spp. was also detected by overlay method. All the 18 isolates of Lactobacillus were used to study the inhibitory activity against $E$. coli and $S$. aureus. In overlay method, out of 18 isolates, $10(55 \%)$ showed the zone of inhibition against the organisms studied as shown in Table I. The inhibitory activity in overlay method was shown only by the isolates which inhibited the growth of E. coli and $S$. aureus in well diffusion assay. Two isolates of L. fermentum (LB-2 and LB-3), two of L. plantarum (LB-8 and LB-16), and one of L. acidophilus (LB-15) showed strong inhibition zone ( $>2 \mathrm{~mm}$ ) as compared to other isolates which showed moderate zone of inhibition. 
Table: I

Inhibitory pattern of Lactobacillus spp. against indicator strains by agar well diffusion assay and overlay method

\begin{tabular}{|c|c|c|c|c|c|}
\hline \multirow[t]{2}{*}{ Sample } & \multirow[t]{2}{*}{ Lactobacillus spp. } & \multicolumn{2}{|c|}{ Escherichia coli } & \multicolumn{2}{|c|}{ Staphylococcus aureus } \\
\hline & & $\begin{array}{c}\text { Agar well } \\
\text { diffusion assay }\end{array}$ & Overlay method & $\begin{array}{c}\text { Agar well } \\
\text { diffusion assay }\end{array}$ & $\begin{array}{l}\text { Overlay } \\
\text { method }\end{array}$ \\
\hline Curd & L. plantarum LB-1 & - & - & - & - \\
\hline Curd & L. fermentum LB-2 & + & ++ & + & ++ \\
\hline Curd & L. fermentum LB-3 & + & ++ & + & ++ \\
\hline Curd & L. plantarum LB-4 & - & - & - & - \\
\hline Curd & L.acidophillus LB-5 & + & + & + & + \\
\hline Curd & L. acidophilus LB-6 & - & - & - & - \\
\hline Curd & L. plantarum LB-7 & - & - & - & - \\
\hline Cheese1 & L. plantarum LB-8 & + & ++ & + & ++ \\
\hline Cheese & L. plantarum LB9 & + & + & + & + \\
\hline Cheese & L. acidophilus LB10 & - & - & - & - \\
\hline $\begin{array}{l}\text { Fermented } \\
\text { milk }\end{array}$ & L. acidophilus LB-11 & + & + & + & + \\
\hline $\begin{array}{l}\text { Fermented } \\
\text { milk }\end{array}$ & L. fermentum LB-12 & - & - & - & - \\
\hline Lassi & L. plantarum LB-13 & + & + & + & + \\
\hline Lassi & L. acidophilus LB-14 & - & - & - & - \\
\hline Butter & L. acidophilus LB-15 & + & + & + & ++ \\
\hline Butter & L. plantarum LB-16 & + & ++ & + & ++ \\
\hline Butter & L. fermentum LB -17 & - & - & - & - \\
\hline Milk & L. plantarum LB-18 & + & + & - & + \\
\hline
\end{tabular}

Degree of inhibition: $+=$ moderate inhibition zone $(<2 \mathrm{~mm}) ;++=$ strong inhibition zone $;(>2 \mathrm{~mm}) ;-=$ no inhibition

The L. fermentum LB-2 and LB-3 showing more inhibitory activity, were isolated from curd sample, the isolates $L$. plantarum LB-8 was isolated from cheese sample whereas $L$. acidophilus LB-15 and LB-16 were isolated from butter sample.

Out of a total of 18 different fermented milk products, 10 Lactobacillus strains (55\%) were found to produce bacteriocin like substance by agar well diffusion test thus inhibiting the growth of $E$. coli and $S$. aureus. The isolates showing zone of inhibition in agar well diffusion assay also inhibited the growth of E. coli and $S$. aureus in overlay method, however the zone of inhibition was more in overlay method. The inhibition was more shown by live bacteria as compared to sterile broth of Lactobacillus 


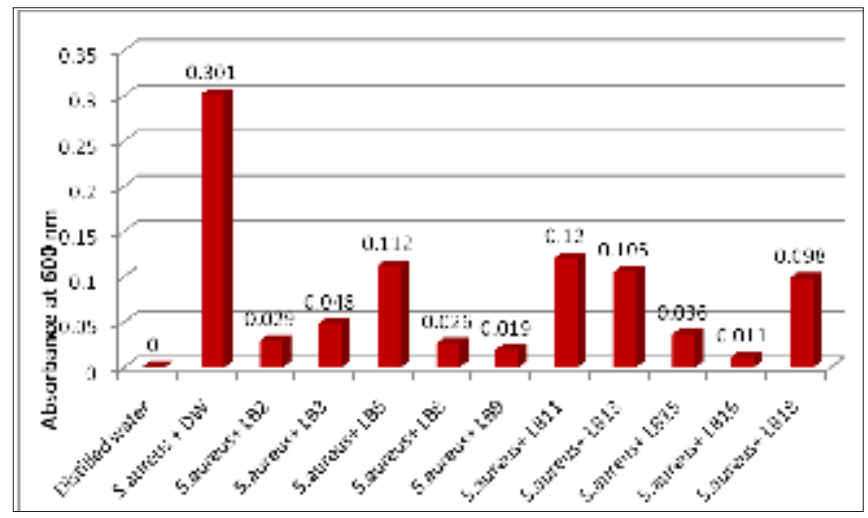

Figure III: Effect of Lactobacillus spp. on biofilm formation by S. aureus

$D W=$ Distilled water, LB Lactobacillus

culture in agar well diffusion assay suggesting other products produced by bacteria during growth like organic acid, hydrogen peroxide, diacetyl and low molecular weight antimicrobial substances could have contributed in inhibition of these pathogens. The antibacterial activity of Lactobacillus strains have been recorded in many studies. Antimicrobial peptides called bacteriocin produced by some microorganisms is one of the alternate methods for controlling pathogenic bacteria. Bacteriocins have attracted much attention due to their ability to act as a biopreservative agents which are used in foods particularly in dairy foods and also in human therapeutics.

The results obtained in our study regarding the production of bacteriocin against human pathogens which causes the gastrointestinal diseases, is in agreement with the work done by other workers. ${ }^{13,14}$

Lactobacillus isolates that showed zone of inhibition in agar well diffusion assay and overlay method were used to determine inhibitory potential against biofilm formation by E.coli and $S$. aureus by microtiter plate method. Different isolates showed different absorbance at $600 \mathrm{~nm}$. Ten bacteriocin producing Lactobacillus spp. isolates were taken for observation of biofilm inhibition produced by E. coli. Maximum absorbance was observed in sample containing E. coli only. The absorbance values were less in the samples where E. coli was mixed with broth containing Lactobacillus spp. All the isolates after incubation showed less absorbance, however 5 samples showed very less absorbance containing $L$. fermentum LB-3 (curd sample), L. plantarum LB-8 (cheese sample), L. acidophilus LB-15 and L. plantarum LB-16 (butter sample). Along with E. coli less absorbance indicated that Lactobacillus spp. broth inhibited the biofilm

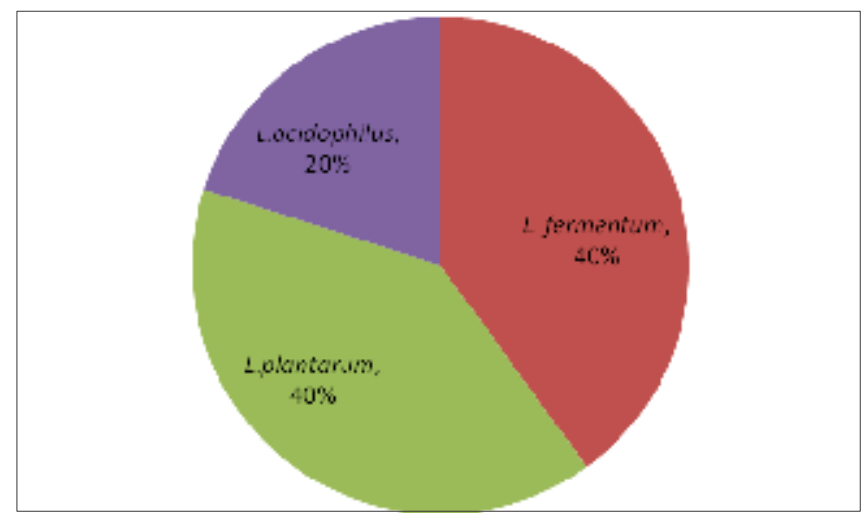

Figure IV: Lactobacillus spp. showing absorbance less than 0.05 in biofilm forming microtiter plate ( $E$ coli)

formation by E. coli.

Similarly ten bacteriocin producing Lactobacillus isolates were also studied for inhibition of biofilm formation by $S$. aureus. All the samples containing Lactobacillus spp. along with the $S$. aureus are shown in Figure III. The sample which contained only $S$. aureus isolates showed maximum absorbance of less than $0.30 \mathrm{~nm}$ whereas absorbance of less than $0.05 \mathrm{~nm}$ was observed in 6 samples which contained Lactobacillus spp. and $S$. aureus. The 6 isolates that showed minimum absorbance were LB-2 (curd sample), LB-3 (curd sample), LB-8 (cheese sample), LB-15 and LB-16 (butter sample). Less absorbance indicates that Lactobacillus spp. isolates inhibits the biofilm formation by S. aureus. Escherichia are human pathogens and are attached with epithelial cells of gastrointestinal tract. They divide rapidly and make biofilm there. These pathogens cause gastrointestinal diseases by secreting toxins. Lactobacillus produces bacteriocin compounds that are proteinaceous peptides.

The isolates showing absorbance even less than 0.05 against $E$. coli were $L$. fermentum $40 \%, L$. plantarum $40 \%$, and L. acidophillus $20 \%$ shown in Figure IV. The isolates showing absorbance even less than 0.05 against S. aureus were L. fermentum 34\%, L. plantarum 50\%, and L. acidophillus 16\% shown in Figure V. Fracchia et $a l^{6}$ observed that Lactobacilli isolated from fresh fruits and vegetables produced biosurfactants and antimicrobial compounds after 5 hours of growth in the mid-exponential phase which significantly $(\mathrm{p}<0.05)$ inhibited the adhesion of two Candida albicans pathogenic biofilm producer strains in pre-coating and co-incubation experiments indicating that the biosurfactant displayed anti-biofilm 


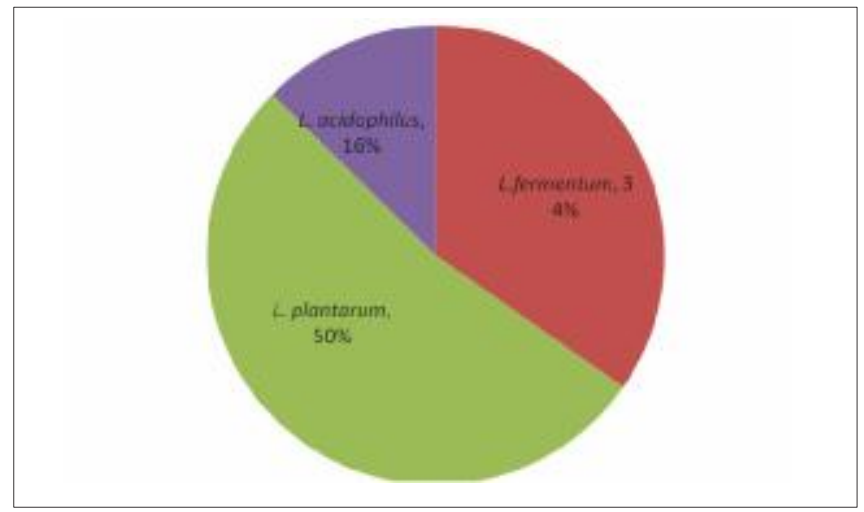

Figure V: Lactobacillus spp. showing absorbance less than 0.05 in biofilmmicrotiter plate (S. aureus)

formation. The mechanism of interference in biofilm formation is due to the release of biosurfactants. ${ }^{15,16}$ Adsorption of biosurfactants to a substratum surface modifies its hydrophobicity, interfering in the microbial adhesion and desorption process. ${ }^{15}$ In that sense the release of biosurfactant by probiotic bacteria in vivo can be considered as a defence weapon against other colonizing strains in the gastrointestinal tract. ${ }^{17}$ In the present study, total 21 fermented dairy products were processed for Lactobacillus spp. isolation. A total of 18 isolates were speciated biochemically of which $45 \%$ of L. plantarum, $33 \%$ of $L$. acidophilus and $22 \%$ of $L$. fermentum. All 18 isolates were processed for antimicrobial activity by agar well diffusion and overlay method against $E$. coli and $S$. aureus. Ten isolates showed inhibitory activity against $E$. coli and $S$. aureus in agar well diffusion assay and overlay method. Of the 10 isolates, 5 isolates also prevented the biofilm formation by these pathogens. The beneficial attributes of lactic acid bacteria have been reported in many studies. The isolates showing inhibitory activities against the pathogens tested can be used as probiotics or as starter culture in food fermentations.

\section{ACKNOWLEDGEMENTS}

The study was carried out by the internal grants of the Institute. We are indebted to the Management of Shaheed Udham Singh Group of Institutes for providing the infrastructure and grants required for conducting the study.

\section{Conflict of interest: None}

\section{REFERENCES}

1. Cross ML. Microbes versus microbes: immune signals generated by probiotics Lactobacilli and their role in protection against microbial pathogens. FEMS Immunol Med Microbiol 2002;34(4):245-53.
2. McFarland LV. Evidence-based review of probiotics for antibioticassociated diarrhea and Clostridium difficile infections. Anaerobe 2009;15(6):274-80.

3. Mattila-Sandholm T, Myllarinen P, Crittenden R, Mogensen G, Fonden R, Saarela M. Technological challenges for future probiotic foods. Int Dairy J 2002;12:173-82.

4. Gupta V, Garg R. Probiotics. Indian J Med Microbiol 2009;27(3):202-9.

5. Savadogo A, Ouattara CAT, Bassole IHN, Traore SA. Bacteriocin and Lactic acid bacteria- a minireview. Afr J Biotechnol 2006;5(9):678-83.

6. Fracchia L, Cavallo M, Allegrone G, Martinitti MG. A Lactobacillus derived biosurfactant inhibits biofilm formation of human pathogenic Candida albicans biofilm producers. In: Current research technology and education Topics in Applied Microbiology and Microbial Biotechnology, A. Mendez- Vilas (Ed). 2010; 827-38.

7. Bogovic-Matijasic B, Rogelj I, Nes IF, Holo H. Isolation and characterization of two bacteriocins of Lactobacillus acidophilus LF221. Appl Microbiol Biotechnol 1998;49:606-12.

8. Cappuccino JG, Sherman N. Microbiology: A laboratory manual. 7th edition. Pearson Education, Inc. and Dorling Kindersley, India. 2009.

9. Tagg JR, McGiven AR. Assay system for bacteriocins. Appl Microbiol 1971;21(5):943.

10. Merritt JH, Kadouri DE, O'Toole GA. Growing and analyzing static biofilms. In: Current Protocol in Microbiology. John Wiley \& Sons, Inc. 2005. p IB.1.1-IB.1.17

11. Jagadeeswari S, Vidya P, Kumar DJM, Balakumaran MD. Isolation and characterization of bacteriocin producing Lactobacillus spp. from traditional fermented foods. Elec J Environ Agricult Food Chem 2010;9(3):575-81.

12. Tambekar DH, Bhutada SA. Studies on antimicrobial activity and characteristics of bacteriocin produced by Lactobacillus strains isolated from milk of domestic animals. The Internet Journal of Microbiology. 2010;8:DOI:10.5580/18b1

13. Anas M, Eddine HJ, Mebrouk K. Antimicrobial activity of Lactobacillus species isolated from Algerian raw goat's milk against Staphylococcus aureus. World J Dairy Food Sci 2008;3(2):39-49.

14. Bhattacharya S, Das A. Study of physical and cultural parameters on the bacteriocins produced by lactic acid bacteria isolated from traditional Indian fermented foods. Am J Food Technol 2010;5(2):111-20.

15. Rodrigues LR, Teixeira JA, van der Mei HC, Oliveira R. Physicochemical and functional characterization of a biosurfactant produced by Lactococcus lactis 53. Colloids Surf B Biointerfaces 2006;49(1):79-86.

16. Gudina EJ, Teixeira JA, Rodrigues LR. Isolation and functional characterization of a biosurfactant produced by Lactobacillus paracasei. Colloids Surf B Biointerfaces 2010;76(1):298-304.

17. vanHoogmoed CG, van der Mei HC, Busscher HJ. The influence of biosurfactants released by S. mitis BMS on the adhesion of pioneer strains and cariogenic bacteria. Biofouling 2004;20(6):261-7. 